See discussions, stats, and author profiles for this publication at: https://www.researchgate.net/publication/3450545

\title{
A Biologically Inspired Biped Locomotion Strategy for Humanoid Robots: Modulation of Sinusoidal Patterns by a Coupled Oscillator Model
}

Article in IEEE Transactions on Robotics · March 2008

DOI: 10.1109/TRO.2008.915457 · Source: IEEE Xplore

4 authors, including:

G. Endo

Tokyo Institute of Technology

254 PUBLICATIONS 2,105 CITATIONS

SEE PROFILE

Some of the authors of this publication are also working on these related projects:

Project INTERACT: Brain-To-Sound Computer Interfaces View project

Project Everyday Activity Science and Engineering (EASE) View project 


\section{Short Papers}

\section{A Biologically Inspired Biped Locomotion Strategy for Humanoid Robots: Modulation of Sinusoidal Patterns by a Coupled Oscillator Model}

Jun Morimoto, Gen Endo, Jun Nakanishi, and Gordon Cheng

\begin{abstract}
Biological systems seem to have a simpler but more robust locomotion strategy than that of the existing biped walking controllers for humanoid robots. We show that a humanoid robot can step and walk using simple sinusoidal desired joint trajectories with their phase adjusted by a coupled oscillator model. We use the center-of-pressure location and velocity to detect the phase of the lateral robot dynamics. This phase information is used to modulate the desired joint trajectories. We do not explicitly use dynamical parameters of the humanoid robot. We hypothesize that a similar mechanism may exist in biological systems. We applied the proposed biologically inspired control strategy to our newly developed human-sized humanoid robot computational brain $(\mathrm{CB})$ and a small size humanoid robot, enabling them to generate successful stepping and walking patterns.
\end{abstract}

Index Terms-Biologically inspired approach, biped walking, central pattern generator, coupled oscillator, humanoid robots.

\section{INTRODUCTION}

Biological systems seem to have a simpler but a more robust locomotion strategy [1], [2] than that of the existing biped walking controllers for humanoid robots. For examples, it was shown in [2] and [3] that the cat locomotion system can generate walking pattern without using higher brain function. An early study of biologically inspired approach to bipedal locomotion [4] suggested that the synchronization property of neural system with periodic sensor inputs plays an important role for robust locomotion control. After this leading study, there is growing interest in the biologically inspired locomotion control utilizing coupled neural oscillators [5]-[8] or using a phase oscillator model with phase reset methods [9], [10]. These studies make use of the foot-contact information or ground reaction forces in exploiting the entrainment property of the neural or phase oscillator model.

Similarly, since biped walking is a periodic movement, it has been suggested that methods to synchronize biped controllers with the robot dynamics are useful to generate stable walking patterns. Several studies designed walking trajectories as a function of a physical variable of the robot (e.g., ankle joint angle) [11]-[13].

However, a neural oscillator model has complex dynamics and many parameters to be selected [4], [5]. Other approaches that has synchronization mechanisms usually require proper gait design [9], [10], [12], [13].

Manuscript received February 1, 2007; revised October 16, 2007. This paper was recommended by Associate Editor S. Ma and Editor H. Arai upon evaluation of the reviewers' comments.

J. Morimoto, J. Nakanishi, and G. Cheng are with the Japan Science and Technology, Saitama 332-0012, Japan. They are also with the Advanced Telecommunication Research (ATR) Computational Neuroscience Laboratories, Kyoto 619-0288, Japan (e-mail: xmorimo@atr.jp; jun@atr.jp; gordon@atr.jp).

G. Endo is with the Department of Mechanical and Aerospace Engineering, Tokyo Institute of Technology, Tokyo 152-8552, Japan (e-mail: gendo@sms. titech.ac.jp).

Color versions of one or more of the figures in this paper are available online at http://ieeexplore.ieee.org.

Digital Object Identifier 10.1109/TRO.2008.915457

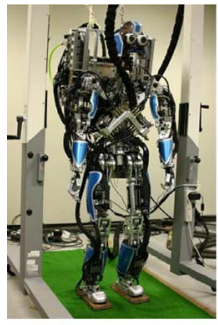

(a)

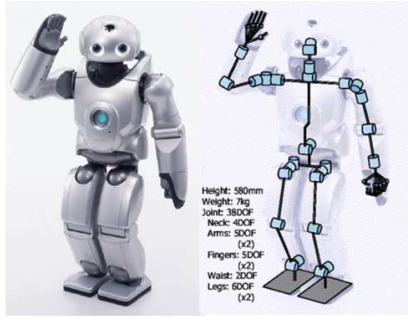

(b)

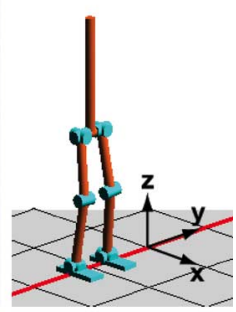

(c)
Fig. 1. (a) Our human-sized hydraulic humanoid robot CB developed by SARCOS [20] of height, $1.59 \mathrm{~m}$ and total weight, $95 \mathrm{~kg}$. (b) Small humanoid robot used in the experiment. (c) Simple 3-D biped simulation model. The biped model has ten DOF, height, $1.59 \mathrm{~m}$ and total weight, $95 \mathrm{~kg}$.

In this study, we undertake the development of a simple but robust biped controller by means of a coupled oscillator system, which is said to exist in vertebrates and is widely referred to as central pattern generator (CPG) [14].

Many biped walking studies have emphasized that humanoid robots have inverted pendulum dynamics, with the top at the center of mass $(\mathrm{COM})$ and the base at the center of pressure (COP), and proposed control strategies to stabilize the dynamics [15]-[19].

We propose using the COP to detect the phase of the inverted pendulum dynamics; 1) we use simple periodic functions (sinusoids) as desired joint trajectories; 2) we show that synchronization of the desired trajectories at each joint with the inverted pendulum dynamics can generate stepping and walking; 3) since our nominal gait patterns are sinusoids, our approach does not need careful design of desired gait trajectories; 4) we use smaller numbers of parameters than that used in the existing neural oscillator approach, and compare to the neural oscillator model, parameters used in our approach can be easily selected since the physical meanings of the parameters are quite simple.

To the best of our knowledge, this study is the first attempt to apply an oscillator model to a human-sized humanoid robot computational brain (CB) [20] [Fig. 1(a)] for biped walking in a real environment. We also apply our method to a small humanoid robot [Fig. 1(b)]. First, we introduce our biologically inspired biped locomotion strategy, which use modulated sinusoidal patterns via a coupled oscillator model, described in Section II. In Section III, we apply our proposed approach to the simulated robot model [see Fig. 1(c)], and also show our experimental results.

\section{MOdUlation OF Sinusoidal PATterns By A COUPLED OSCILLATOR MODEL}

Our biped control approach uses a coupled phase oscillator model [21] to modulate sinusoidal patterns. The aim of using the coupled phase oscillator model is to synchronize periodic patterns generated by the controller with the dynamics of the robot.

We also show a strategy to design a nominal desired joint angle. One of the simplest ways to generate the periodic pattern at each joint is using only one sinusoidal basis function to represent the desired joint angle. By using only one sinusoidal basis function at each joint, we have smallest numbers of parameters to represent periodic patterns at each joint. We introduce our stepping and walking controllers that use the desired joint angle represented by the sinusoidal function. 


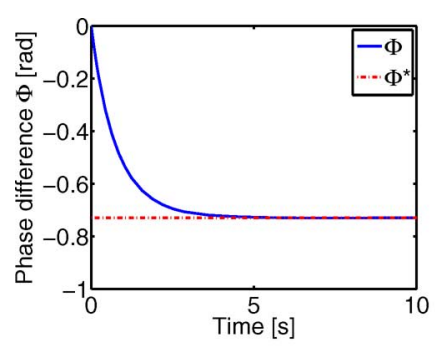

(a)

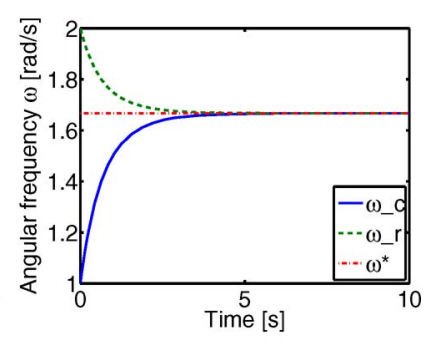

(b)
Fig. 2. Typical time profile of the coupled oscillator system. (a) Phase difference $\Phi=\phi_{r}-\phi_{c}$. The phase difference converged to the analytically derived value $\Phi^{*}$ depicted by dash dot line. (b) Angular frequencies $\omega_{r}$ and $\omega_{c}$. The angular frequency of each oscillator converged to the analytically derived compromise frequency $\omega^{*}$.

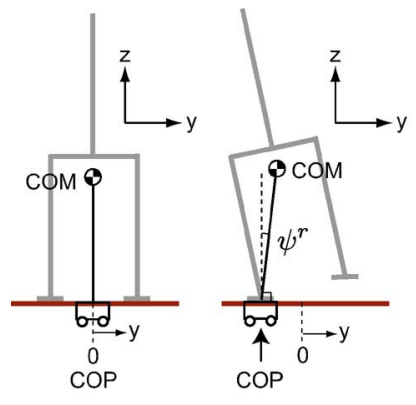

(a)

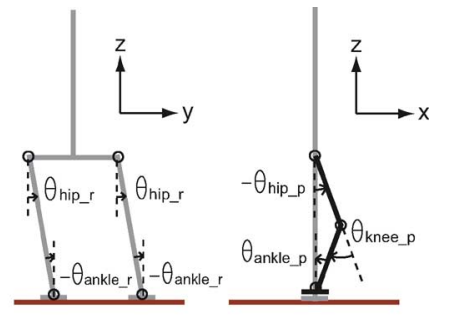

(b)
Fig. 3. (a) Inverted pendulum model represented by the COP and the COM. (b) Notations for joint angles.

\section{A. Coupled Oscillator Model}

Here, we consider the behavior of the dynamics of the phase of a biped controller $\phi_{c}$ and the phase of the robot dynamics $\phi_{r}$ as

$$
\begin{aligned}
& \dot{\phi}_{c}=\omega_{c}+K_{c} \sin \left(\phi_{r}-\phi_{c}\right) \\
& \dot{\phi}_{r}=\omega_{r}+K_{r} \sin \left(\phi_{c}-\phi_{r}\right)
\end{aligned}
$$

where $\omega_{c}>0$ and $\omega_{r}>0$ are natural frequencies of the controller and the robot dynamics, and $K_{c}$ and $K_{r}$ are positive coupling constants. We can find two fixed points if $\left|\omega_{c}-\omega_{r}\right|<K_{c}+K_{r}$. There is no fixed point if $\left|\omega_{c}-\omega_{r}\right|>K_{c}+K_{r}$. A saddle-node bifurcation occurs when $\left|\omega_{c}-\omega_{r}\right|=K_{c}+K_{r}$.

If $\left|\omega_{c}-\omega_{r}\right|<K_{c}+K_{r}$, the oscillators run with the phase difference $\Phi^{*}=\phi_{r}-\phi_{c}=\sin ^{-1}\left(\left(\omega_{r}-\omega_{c}\right) /\left(K_{c}+K_{r}\right)\right)$ and the compromise frequency $\omega^{*}=\left(K_{r} \omega_{c}+K_{c} \omega_{r}\right) /\left(K_{c}+K_{r}\right)$ when they are entrained [21].

We showed the typical time profile of phase difference $\Phi=\phi_{c}-$ $\phi_{r}$ and angular frequencies $\omega_{c}$ and $\omega_{r}$ in Fig. 2. Although the biped dynamics usually cannot be represented by the simple phase dynamics (2), we can still detect the phase from the robot dynamics as described in Section II-B. Then, we use (1) to adjust the phase of the controller under the assumption that the phase dynamics detected from the biped dynamics keeps property similar to (2).

\section{B. Phase Detection from the Robot Dynamics}

As previous studies have pointed out, controlling the inverted pendulum dynamics represented by the COM and the COP [Fig. 3(a)] is a major issue in controlling biped robots. We consider the inverted pendulum dynamics on a lateral plane that has a four-dimensional state space $\mathbf{x}=\left(y, \dot{y}, \psi^{r}, \dot{\psi}^{r}\right)$, which is depicted in Fig. 3(a).

To detect the phase from the inverted pendulum dynamics, we project the four-dimensional state space to a two-dimensional state space. Then, convert the two-dimensional state space to the phase space by using the inverse tangent, which is widely used as a phase detector for radio wave decoding.

In this study, we consider the COP $y$ and the velocity of the COP $\dot{y}$ as the variables in the two-dimensional state space, since detecting these values by force sensors on soles is easy for our real robots. Therefore, we detect the phase as

$$
\phi_{r}(\mathbf{x})=-\arctan \left(\frac{\dot{y}}{y}\right) .
$$

\section{Simplified COP Detection}

The COP depends on a coordinate system, and we need a kinematic model to detect COP. Alternatively, we use an approximate COP as

$$
y=\frac{y_{\text {foot }}^{l} F_{z}^{l}+y_{\text {foot }}^{r} F_{z}^{r}}{F_{z}^{r}+F_{z}^{l}}
$$

where $F_{z}^{l}$ and $F_{z}^{r}$ represent the left and right ground reaction force, respectively, and $y_{\text {foot }}^{l}$ and $y_{\text {foot }}^{r}$ are the lateral position of each foot. We assume that feet are symmetrically placed, i.e., $-y_{\text {foot }}^{l}=y_{\text {foot }}^{r}$. Because we only use this COP to detect the phase of the robot dynamics $\phi_{r}(\mathbf{x})$ in (3), the scale of the foot position $y_{\text {foot }}^{l}$ and $y_{\text {foot }}^{r}$ can be arbitrary. We simply set $y_{\text {foot }}^{l}=-y_{\text {foot }}^{r}=1.0 \mathrm{~m}$. This simplified that the COP detection does not require the kinematic model.

When we derive the COP from the sensor input, we use a low-pass filter to eliminate the sensor noise from force sensors on soles.

Note that when the COP comes to an edge of a sole, the detected $\phi_{r}(\mathbf{x})$ may have discontinuous change because of discontinuous change of the velocity of the COP [see (3)]. However, because we apply the low-pass filter to the force sensor input, the filtered velocity of the $\mathrm{COP}$ do not have such undesirable discontinuous change.

\section{Phase Coordination}

In this study, we use two oscillators with phases $\phi_{c_{-} i}$, where $i=$ 1,2. We introduce coupling between the oscillators and the phase of the robot dynamics $\phi_{r}(\mathbf{x})$ to regulate the desired phase relationship between the oscillators as in (1) as

$$
\dot{\phi}_{c_{-} i}=\omega_{c}+K_{c} \sin \left(\left(\phi_{r}(\mathbf{x})-\alpha_{i}\right)-\phi_{c_{-} i}\right)
$$

where $\alpha_{i}$ is the desired phase difference. We use two different phase differences $\left\{\alpha_{1}, \alpha_{2}\right\}=\{-1 / 2 \pi, 1 / 2 \pi\}$ to make symmetric patterns for a stepping movement by left and right limbs (see Section II-E2). Two parameters $\left\{\omega_{c}, K_{c}\right\}$ need to be selected to define the phase oscillator dynamics.

We empirically found that the natural frequency of a linear pendulum with length $l$ can be a good candidate for the natural frequency of the controller as $\omega_{c}=\sqrt{g / l}$, where $g$ denotes the acceleration due to the gravity and $l$ denotes the height of the COM when a biped stands straight.

By considering the insight from the oscillator dynamics (1) and (2), we need to use a sufficiently large coupling constant $K_{c}$ to satisfy $\left|\omega_{c}-\omega_{r}\right|<K_{c}+K_{r}$ for keeping fixed points.

\section{E. Stepping Controller for Lateral Movement}

1) Side-to-Side Controller for Lateral Movement: First, we introduce a controller to generate a side-to-side movement. We use the hip 
joints $\theta_{\text {hip_r }}$ and the ankle joints $\theta_{\text {ankle_r }}[$ Fig. 3(b)] for the movement. Desired joint angles for each joint are

$$
\begin{gathered}
\theta_{\text {hip_r }}^{d}\left(\phi_{c}\right)=A_{r} \sin \left(\phi_{c}\right)+\bar{\theta}_{r} \\
\theta_{\text {ankle_r }}^{d}\left(\phi_{c}\right)=-A_{r} \sin \left(\phi_{c}\right)-\bar{\theta}_{r}
\end{gathered}
$$

where $A_{r}$ 's are the amplitudes of a sinusoidal function for side-to-side movements at the hip and the ankle joints, and we use an oscillator with the phase $\phi_{c}=\phi_{c_{-} 1}$. The variable $\bar{\theta}_{r}$ defines the rest posture of the hip and ankle joints. Two parameters $\left\{A_{r}, \bar{\theta}_{r}\right\}$ need to be selected for the side-to-side controller.

2) Vertical Foot Movement to Make Clearance: To achieve foot clearance, we generate a vertical movement of the feet [Fig. 3(b)] by using simple sinusoidal trajectories as

$$
\begin{aligned}
\theta_{\text {hip_p }}^{d}\left(\phi_{c}\right) & =A_{p} \sin \left(\phi_{c}\right)+\bar{\theta}_{p} \\
\theta_{\text {knee_p }}^{d}\left(\phi_{c}\right) & =-2 A_{p} \sin \left(\phi_{c}\right)-2 \bar{\theta}_{p} \\
\theta_{\text {ankle_p }}^{d}\left(\phi_{c}\right) & =-A_{p} \sin \left(\phi_{c}\right)-\bar{\theta}_{p}
\end{aligned}
$$

where $A_{p}$ is the amplitude of a sinusoidal function to achieve the foot clearance and $\bar{\theta}_{p}$ defines the rest posture of the hip, knee, and ankle joints. We use the oscillator with phase $\phi_{c}=\phi_{c_{-} 1}$ for the right limb movement and use the oscillator with phase $\phi_{c}=\phi_{c_{-} 2}$, which has a phase difference $\phi_{c_{-} 2}=\phi_{c_{-} 1}+\pi$, for the left limb movement. Two parameters $\left\{A_{p}, \bar{\theta}_{p}\right\}$ need to be selected for the controller of the vertical foot movement.

Because the COP is modulated by the joint angle trajectories, the phase of the controller $\phi_{c}$ affects the phase of the robot $\phi_{r}(\mathbf{x})$ in (3), through the controllers introduced in (6)-(10).

\section{F. Biped Walking Controller with Additional Sinusoids}

For our biped walking controller, we introduce two additional oscillators $\phi_{c_{-} i}^{p}$, where $i=1,2$. We then consider phase dynamics

$$
\dot{\phi}_{c_{-} i}^{p}= \begin{cases}\omega_{c}+K_{c}^{p} \sin \left(\left(\phi_{r}(\mathbf{x})-\alpha_{i}^{p}\right)-\phi_{c_{-} i}^{p}\right) & \text { (single support) } \\ 0 & \text { (double support). }\end{cases}
$$

We set the dynamics of the oscillator $\dot{\phi}_{c_{-} i}^{p}=0$ during the double support phase, so that we can prevent the internal force generated by the friction between the ground and the soles, while we keep using the phase dynamics (5). We use two different phase differences $\left\{\alpha_{1}^{p}, \alpha_{2}^{p}\right\}=$ $\{0.0, \pi\}$ to make symmetric patterns for a forward movements by left and right limbs. We empirically figured out that we can generate biped walking by setting the coupling constant as $K_{c}^{p}=K_{c} / 2$.

To walk forward, the biped needs to make forward step. To make forward step at proper timing, we introduce an additional sinusoidal trajectory that has $1 / 2 \pi$ phase difference from the sinusoidal trajectories for the stepping movement

$$
\begin{aligned}
\theta_{\text {hip } \_s}^{d}\left(\phi_{c}^{p}\right) & =A_{h_{-s}} \sin \left(\phi_{c}^{p}\right) \\
\theta_{\text {ankle_s }}^{d}\left(\phi_{c}^{p}\right) & =-A_{a_{\_} s} \sin \left(\phi_{c}^{p}\right)
\end{aligned}
$$

where $A_{h_{-}}$and $A_{a_{-} s}$ are amplitudes of sinusoidal functions at the hip and the ankle joints for biped walking. We use the phase $\phi_{c}^{p}=\phi_{c_{-} 1}^{p}$ that has $1 / 2 \pi$ phase difference with $\phi_{c_{-}}$, that is, the phase of the oscillator for the lateral movement for right limb, and use the phase $\phi_{c}^{p}=\phi_{c_{-} 2}^{p}$ that has $\pi$ phase difference with $\phi_{c_{-} 1}^{p}$. The desired nominal trajectories for hip and ankle pitch joints in (8) and (10) become

$$
\begin{aligned}
\theta_{\text {hip_p }}^{d}\left(\phi_{c}, \phi_{c}^{p}\right) & \leftarrow \theta_{\text {hip_p }}^{d}\left(\phi_{c}\right)+\theta_{\text {hip_s }}^{d}\left(\phi_{c}^{p}\right) \\
\theta_{\text {ankle_p }}^{d}\left(\phi_{c}, \phi_{c}^{p}\right) & \leftarrow \theta_{\text {ankle_p }}^{d}\left(\phi_{c}\right)+\theta_{\text {ankle_s }}^{d}\left(\phi_{c}^{p}\right) .
\end{aligned}
$$

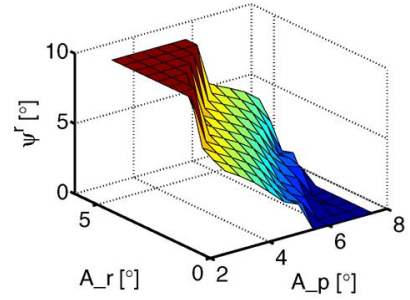

(a)

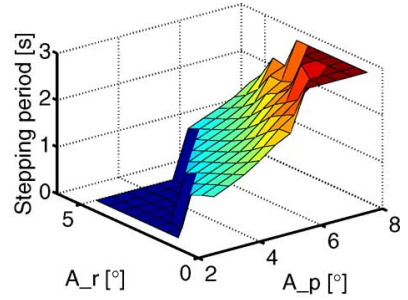

(b)
Fig. 4. Comparison of using different amplitude parameters. (a) Pendulum angle $\psi^{r}$ in Fig. 3(a). Region that has the value $\psi^{r}=\mathbf{1 0}$ represents that the robot cannot make single support phase with the corresponding parameter selection. Region that has the value $\psi^{r}=\mathbf{0}$ represents that the robot falls over with the corresponding parameter selection. (b) Stepping period. Region that has the value 0 s represents that the robot cannot make single support phase with the corresponding parameter selection. Region that has the value $3 \mathrm{~s}$ represents that the robot falls over with the corresponding parameter selection.

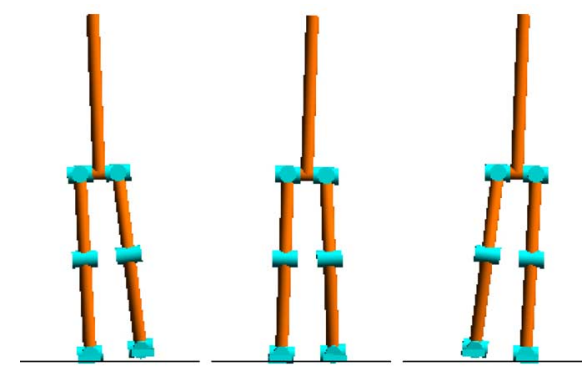

(a)

(b)

(c)

Fig. 5. Stepping movement using coupled oscillator. (a) $t=0.0 \mathrm{~s}$. (b) $t=$ 0.4 s. (c) $t=0.8 \mathrm{~s}$.

Two parameters $\left\{A_{h_{-} s}, A_{a_{-} s}\right\}$ need to be selected for the controller for forward movements.

\section{Simulation AND EXPERIMENTAL RESUlt}

We applied our proposed method to a simple three-dimensional biped robot model [Fig. 1(c)], our human-sized humanoid robot $\mathrm{CB}$ [Fig. 1(a)], and the small humanoid robot [Fig. 1(b)].

\section{A. Stepping Movement}

1) Application To the Simulated Biped Model: We applied our proposed method to the biped robot model.

As we proposed in Section II-D, the natural frequency of the controller is set as $\omega_{c}=\sqrt{g / l}=3.6 \mathrm{rad} / \mathrm{s}$ and the coupling constant is set as $K_{c}=10.0$. Then, we compared the different parameter settings for the amplitude $A_{r}$ in (6) and (7) and $A_{p}$ in (8)-(10).

Fig. 4 shows results of the comparison. Using large $A_{p}$ with small $A_{r}$ results in falling over. On the other hand, using small $A_{p}$ with large $A_{r}$ cannot make a single support phase. By comparing Fig. 4(a) and (b), the stepping movement that has smaller pendulum angle $\psi^{r}$ tends to have a larger stepping period.

A proper combination of the parameters $A_{r}=2.5^{\circ}$ and $A_{p}=5.0^{\circ}$, which can make the stepping movement without falling over, generated a stepping movement with period $1.4 \mathrm{~s}$. Equivalently, the average angular frequency $\dot{\phi}_{c}^{\text {av }}=2 \pi / T$ of the stepping movement was $\dot{\phi}_{c}^{\text {av }}=$ $4.5 \mathrm{rad} / \mathrm{s}$, where $T$ is a stepping period. Fig. 5 shows a successful stepping of the simulated biped model. 


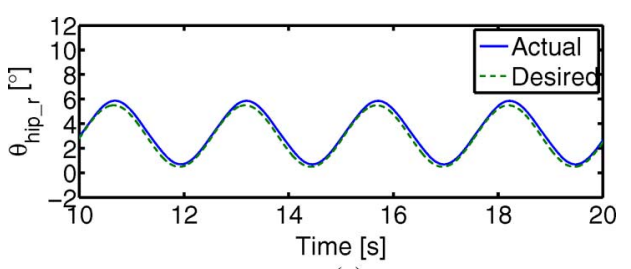

(a)

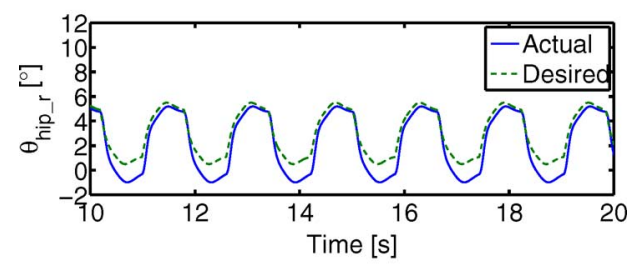

(b)

Fig. 6. Generated desired and actual trajectory at hip joint. (a) Without using coupled oscillator. (b) Using coupled oscillator. Large tracking error appeared during the single support phase due to not using a very large servo gain. The result shows that our proposed method does not require accurate tracking performance.

To show how the coupled oscillator model in (1) and (2) worked with the biped robot model, we tested a different controller with a different natural frequency $\omega_{c}=2.5 \mathrm{rad} / \mathrm{s}$. Although the natural frequency was different, the modulated averaged frequency $\dot{\phi}_{c}^{\text {av }}=3.9 \mathrm{rad} / \mathrm{s}$ was much closer to the previous averaged frequency $\dot{\phi}_{c}^{\text {av }}=4.5 \mathrm{rad} / \mathrm{s}$ than the selected natural frequency $\omega_{c}=2.5 \mathrm{rad} / \mathrm{s}$.

By considering the compromise frequency $\omega^{*}$ introduced in Section II-A, this result indicates that the current coupling constant $K_{c}=10.0$ is large enough to make the controller frequency close to the natural frequency of the robot dynamics.

Fig. 6 shows trajectories of desired and actual hip joint angles $\theta_{\text {hip _r }}^{d}$ and $\theta_{\text {hip_r }}$ [see Fig. 3(b)]. Fig. 6(a) represents the result of not using a coupled oscillator model. The desired trajectory is the original simple sinusoidal trajectory.

Fig. 6(b) represents the result of using a coupled oscillator model. This modulated trajectory made the stepping movement possible. A large tracking error appeared during the single support phase due to not using a very large servo gain. This result shows that our proposed method does not require an accurate tracking performance. The desired trajectory is modulated by the coupling with the phase of the robot dynamics $\phi_{r}(\mathbf{x})$.

2) Application to Human-Sized Humanoid Robot: The proposed stepping method was applied to the human-sized humanoid robot CB.

The natural frequency of the controller is selected as $\omega_{c}=3.14 \mathrm{rad} / \mathrm{s}$ and a sufficiently large coupling constant is selected as $K_{c}=9.4$ by following the parameter selection approach introduced in Section II-D.

We empirically figured out proper amplitude parameters $A_{-r}=3.0^{\circ}$ and $A_{p}=3.5^{\circ}$, which can generate a stepping movement.

Fig. 7 shows a successful stepping of the human-sized humanoid robot.

3) Application to the Small Humanoid Robot: We applied the proposed stepping method to the small humanoid robot.

The natural frequency of the controller is selected as $\omega_{c}=6.28 \mathrm{rad} / \mathrm{s}$ and a sufficiently large coupling constant is selected as $K_{c}=9.4$ by following the parameter selection approach introduced in Section II-D.

We empirically figured out proper amplitude parameters $A_{-r}=7.5^{\circ}$ for hip, $A_{-r}=2.5^{\circ}$ for ankle, and $A_{p}=3.5^{\circ}$, which can generate a stepping movement.

Fig. 8 shows a successful stepping of the small humanoid robot.
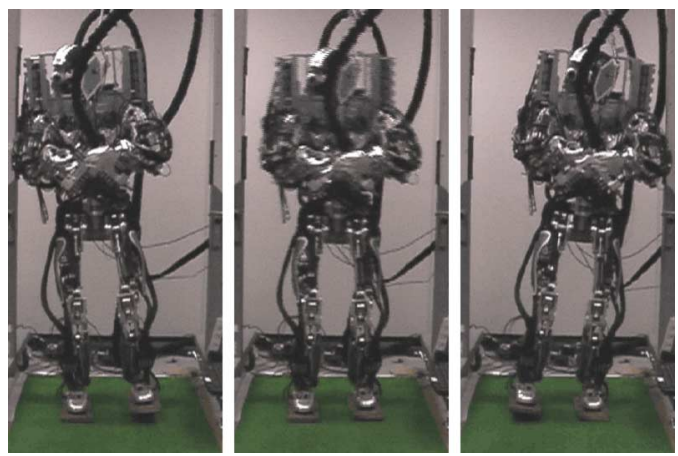

Fig. 7. Successful stepping of our human-sized humanoid robot CB.
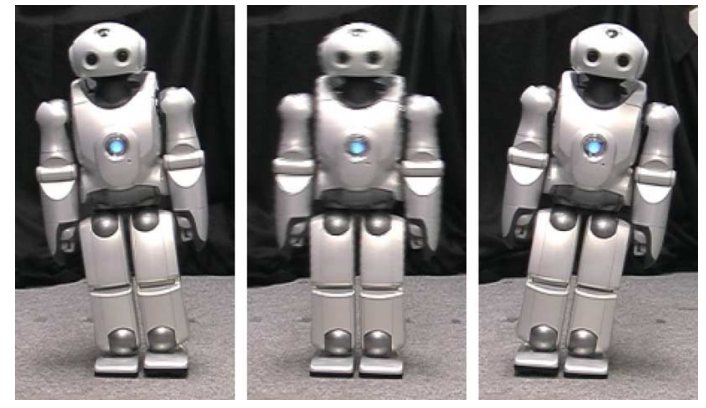

Fig. 8. Successful stepping of the small humanoid robot.

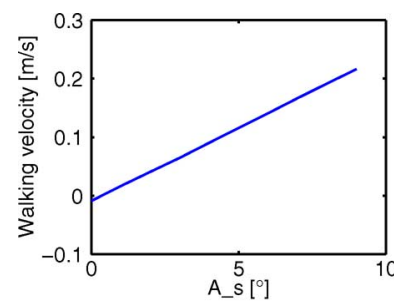

(a)

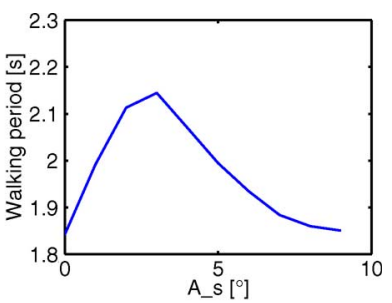

(b)
Fig. 9. Comparison with different amplitude parameters $A_{s}$ in (12) and (13). The biped model falls over with the parameter $A_{s}>9.0^{\circ}$. (a) Walking speed. (b) Walking period.

\section{B. Biped Walking}

1) Application to Simple Biped Model: We applied our proposed method to generate walking movements by using the simulated biped model.

The same parameters as the stepping controller for the natural frequency of the controller $\omega_{c}=3.6 \mathrm{rad} / \mathrm{s}$ and the coupling constant $K_{c}=10.0$ are used. We empirically figured out proper amplitude parameters $A_{r}=2.5^{\circ}$ and $A_{p}=6.0^{\circ}$ for the walking task.

We compared different amplitude parameters $A_{s}$ by setting $A_{h_{-s}}=$ $A_{s}$ in (12) and $A_{a_{-} s}=A_{s} / 2$ in (13).

Fig. 9 shows the results of the comparison. Walking velocity was linearly increased according to the increase of the amplitude parameter $A_{s}$. This is one of the good properties of the proposed walking controller, since we can easily select the amplitude parameter to achieve the desired walking velocity.

On the other hand, the walking period did not show a monotonic change according to the increase of the amplitude $A_{s}$ as in Fig. 9(b). Walking velocity can be increased by either increasing walking step 


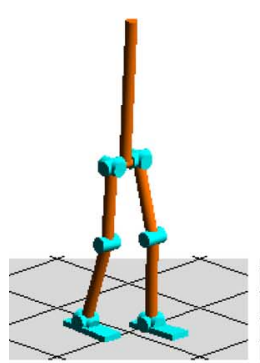

(a)

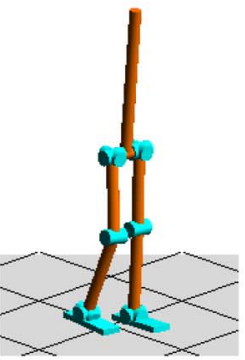

(b)

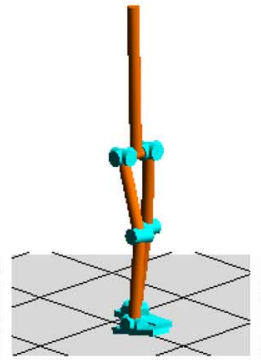

(c)

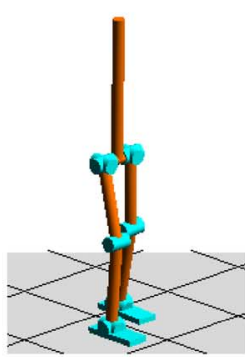

(d)

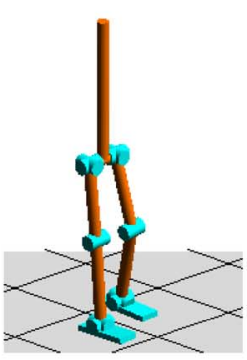

(e)

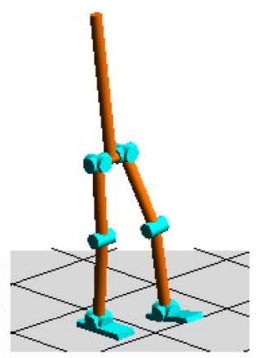

(f)

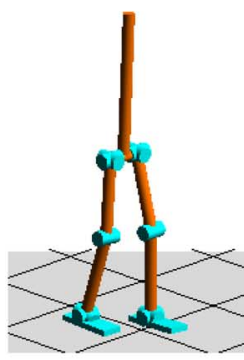

(g)

Fig. 10. Successful walking pattern using simulated biped robot model. Walking speed is $0.22 \mathrm{~m} / \mathrm{s}$. (a) $t=0.0 \mathrm{~s}$. (b) $t=0.30 \mathrm{~s}$. (c) $t=0.57 \mathrm{~s}$. (d) $t=0.93 \mathrm{~s}$. (e) $t=1.23 \mathrm{~s}$. (f) $t=1.53 \mathrm{~s}$. (g) $t=1.87 \mathrm{~s}$.
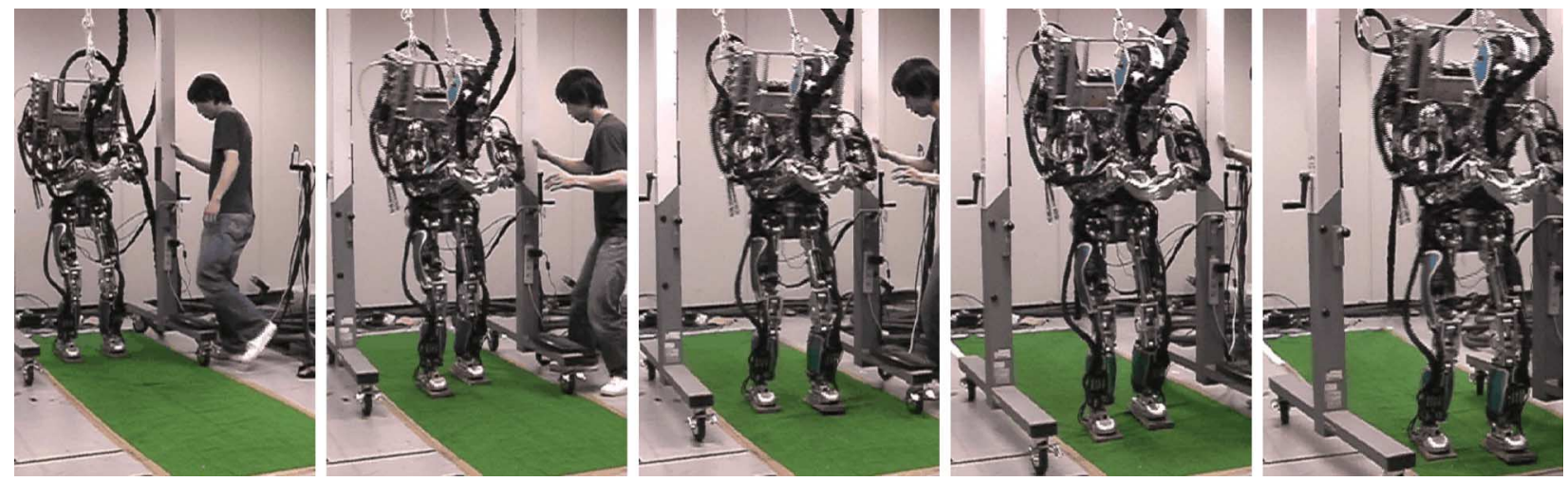

Fig. 11. Successful walking pattern of our human-sized humanoid robot CB.
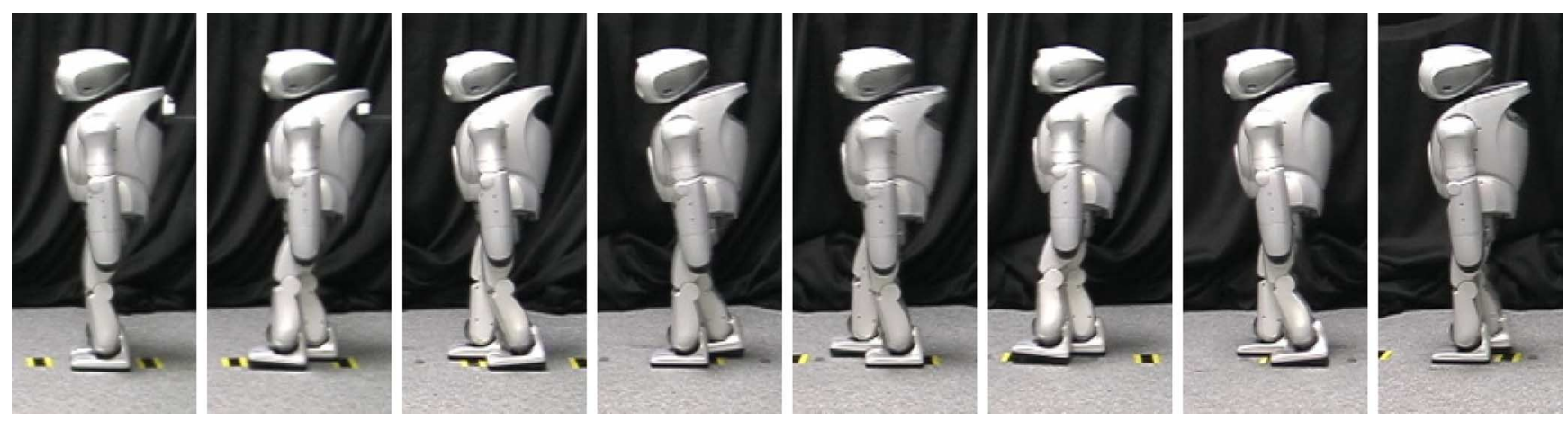

Fig. 12. Successful walking pattern of the small humanoid robot. Knees are stretched during walking.

or decreasing walking period (increased walking frequency). We can see that the walking controller used different strategies to increase the walking speed with different amplitude $A_{s}$.

Fig. 10 shows the successful walking pattern generated by our control approach. We showed that the simulated robot model could walk by only using the simple sinusoidal trajectory, which is composed of at most only two sinusoidal basis functions at each joint, modulated by the detected phase from the COP.

2) Application to Human-Sized Humanoid Robot: We applied our proposed walking method to the humanoid robot CB.

The same parameters as the stepping controller for the natural frequency of the controller $\omega_{c}=3.14 \mathrm{rad} / \mathrm{s}$, the coupling constant $K_{c}=9.4, A_{r}=3.0^{\circ}$, and $A_{p}=3.5^{\circ}$ are used.

We empirically figured out proper amplitude parameters $A_{h_{-} s}=$ $4.0^{\circ}$ and $A_{a_{-} s}=2.0^{\circ}$ for the walking task.

Fig. 11 shows the successful walking pattern of our humanoid robot. Our proposed method was able to generate successful walking patterns even in the real environment.
Note that the black tube from the top of each photo in Fig. 11 is a hydraulic cable.

3) Application to the Small Humanoid Robot: We applied our proposed walking method to the small humanoid robot.

The same parameters as the stepping controller for the natural frequency of the controller $\omega_{c}=6.28 \mathrm{rad} / \mathrm{s}$, the coupling constant $K_{c}=9.4$, amplitudes $A_{-r}=7.5^{\circ}$ for hip, $A_{-r}=2.5^{\circ}$ for ankle, and $A_{p}=3.5^{\circ}$ are used.

We empirically figured out proper amplitude parameters $A_{h_{-} s}=$ $12.0^{\circ}$ and $A_{a_{-} s}=8.0^{\circ}$ for the walking task.

Fig. 12 shows the successful walking pattern of the small humanoid robot. Our proposed method was able to generate successful walking patterns for robots of different sizes. Because our method to design a biped walking controller does not suffer from singularity problem at knee joints that comes from using inverse kinematics, knees can be straighten during walking.

We calculated the ratio between the single support phase and the double support phase. The ratio was around $\left(T_{\text {double }}\right) /\left(T_{\text {double }}+\right.$ 

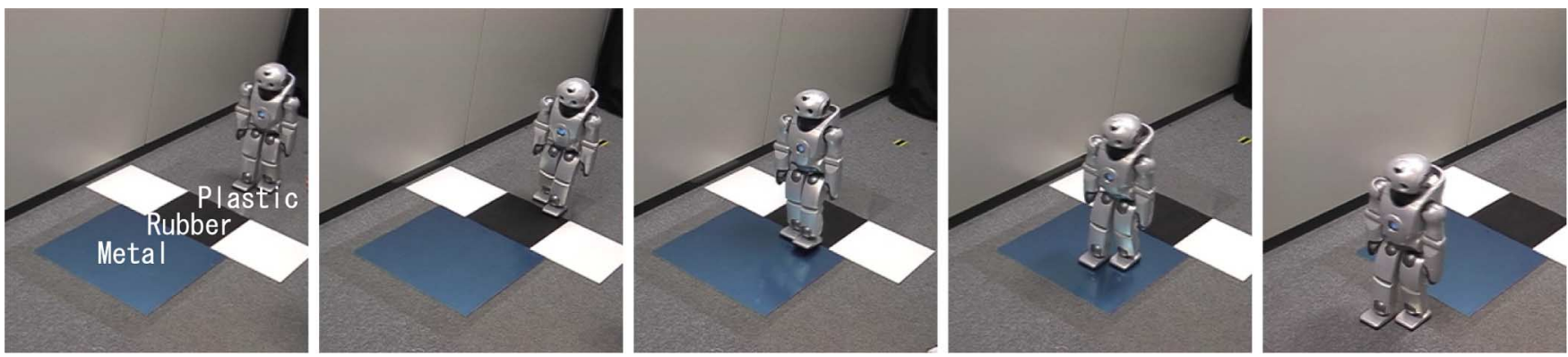

Fig. 13. Successful walking pattern of the small humanoid robot over four different surfaces: 1) Carpet. 2) Plastic sheet. 3) Rubber sheet. 4) Metal sheet.

$\left.T_{\text {single }}\right) \times 100 \%=25 \%$, where $T_{\text {double }}$ is a period of time of the double support phase and $T_{\text {single }}$ is a period of time of the single support phase in a walking cycle. This ratio is similar to the ratio for adult human biped walking $20-25 \%$ that depends on the age of the human subject [22], [23]. This ratio is achieved by our proposed controller without using predesigned trajectories of the COP.

We also tested the robustness of our biped controller by using four different ground surfaces with different frictions. Each surface also has different height. The four surfaces include carpet with $0.0 \mathrm{~mm}$ (base level), plastic sheet with $2.0 \mathrm{~mm}$, rubber sheet with $3.5 \mathrm{~mm}$, and metal sheet with $3.0 \mathrm{~mm}$. Fig. 13 shows successful results of walking over the different surfaces without changing any parameter of the biped controller.

\section{DISCUSSION}

In this paper, we presented a biologically inspired biped locomotion strategy. Our method proposed the utilization of the COP position and velocity to detect the phase of the lateral robot dynamics. Evidences in biological locomotion studies support a part of our paper [1], [24].

The detected phase of the robot dynamics was used to modulate sinusoidal joint trajectories. The modulated trajectories enabled our robots to generate successful stepping and walking patterns. Because the angular frequency in (5) is continuously changing during stepping and walking, not only the frequency of the controller changes toward the resonant frequency and excites the robot dynamics, but also the time course of the sinusoidal patterns are modulated.

We successfully applied our proposed control approach to the newly developed human-sized humanoid robot $\mathrm{CB}$ and a small humanoid robot.

In the future, we will consider using optimization methods such as reinforcement learning or dynamic programming [25], [26] to acquire a nonlinear feedback controller in order to increase the robustness of the walking controller.

\section{APPENDIX}

Here, we describe our simulation setups. To follow the desired trajectories, the torque output at each joint is given by a PD servo controller

$$
\boldsymbol{\tau}=\mathbf{K}_{p}\left(\boldsymbol{\theta}^{d}\left(\phi_{c}\right)-\boldsymbol{\theta}\right)+\mathbf{K}_{d}\left(\dot{\boldsymbol{\theta}}^{d}\left(\phi_{c}\right)-\dot{\boldsymbol{\theta}}\right)
$$

where $\boldsymbol{\theta}^{d}\left(\phi_{c}\right) \in \boldsymbol{R}^{10}$ is the target joint angle vector, $\mathbf{K}_{p}$ denotes the position gain matrix, and $\mathbf{K}_{d}$ denotes the velocity gain matrix. Each element of the diagonal position gain matrix $\mathbf{K}_{p}$ is set to 3000 and each element of the diagonal velocity gain matrix $\mathbf{K}_{d}$ is set to 100 .
We used the fourth-order Runge-Kutta method with a time step of $\Delta t=0.0003 \mathrm{~s}$ to numerically integrate the biped dynamics.

The vertical ground reaction force $f_{z}$ is simulated by a springdumper model given by

$$
f_{z}=-k_{p}^{z} z_{c p}-k_{d}^{z} \dot{z}_{c p}
$$

where $k_{p}^{z}=30000$ is the spring gain, $k_{d}^{z}=1000$ is the dumper gain, and $z_{c p}$ denotes the vertical position of a contact point.

The ground reaction force for horizontal directions $f_{x}$ and $f_{y}$ are simulated by viscous friction

$$
\begin{aligned}
& f_{x}=-k_{d}^{x} \dot{x}_{c p} \\
& f_{y}=-k_{d}^{y} \dot{y}_{c p}
\end{aligned}
$$

where $k_{d}^{x}=2500$ and $k_{d}^{y}=2500$ are dumper gains. $x_{c p}$ and $y_{c p}$ are the horizontal positions of a contact point.

\section{ACKNOWLEDGMENT}

The authors would like to thank Sony Corporation for allowing them the opportunity to use the small humanoid robot. They also would like to thank Darrin Bentivegna for helping them to set up initial experiments. We also thank M. Kawato, C. G. Atkeson, S. Miyakoshi, S. Hyon for helpful discussion.

\section{REFERENCES}

[1] T. A. McMahon, Muscles, Reflexes, and Locomotion. Princeton, NJ: Princeton Univ. Press, 1984.

[2] S. Grillner, "Locomotion in vertebrates: Central mechanisms and reflex Interaction," Phys. Rev., vol. 55, pp. 367-371, 1975.

[3] M. L. Shik and G. N. Orlovsky, "Neurophysisology of locomotion automatism," Phys. Rev., vol. 56, pp. 465-501, 1976.

[4] G. Taga, Y. Yamaguchi, and H. Shimizu, "Self-organized control in bipedal locomotion by neural oscillators in unpredictable environment," Biol. Cybern., vol. 65, pp. 147-159, 1991.

[5] S. Miyakoshi, G. Taga, Y. Kuniyoshi, and A. Nagakubo, "Threedimensional bipedal stepping motion using neural oscillators-Towards humanoid motion in the real world," in IEEE/RSJ Int. Conf. Intell. Robots Syst., Victoria, Canada, 1998, vol. 1, pp. 84-89.

[6] G. Endo, J. Morimoto, J. Nakanishi, and G. Cheng, "An empirical exploration of a neural oscillator for biped locomotion control," in Proc. IEEE 2004 Int. Conf. Robot. Autom., New Orleans, LA, pp. 3063-3042.

[7] G. Endo, J. Nakanishi, J. Morimoto, and G. Cheng, "Experimental studies of a neural oscillator for biped locomotion with QRIO," in IEEE Int. Conf. Robot. Autom., Barcelona, Spain, 2005, pp. 598-603.

[8] Y. Fukuoka and H. Kimura, "Adaptive dynamic walking of a quadruped robot on irregular terrain based on biological concepts," Int. J. Robot. Res., vol. 22, no. 2, pp. 187-202, 2003. 
[9] K. Tsuchiya, S. Aoi, and K. Tsujita, "Locomotion control of a biped locomotion robot using nonlinear oscillators," in Proc. IEEE/RSJ Int. Conf. Intell. Robots Syst., Las Vegas, NV, 2003, pp. 17451750 .

[10] J. Nakanishi, J. Morimoto, G. Endo, G. Cheng, S. Schaal, and M. Kawato, "Learning from demonstration and adaptation of biped locomotion," Robot. Auton. Syst., vol. 47, pp. 79-91, 2004

[11] S. Hyon and T. Emura, "Symmetric walking control: Invariance and global stability," in IEEE Int. Conf. Robot. Autom., Barcelona, Spain, 2005, pp. 1456-1462.

[12] M. Doi, Y. Hsegawa, and T. Fukuda, "Passive trajectory control of the lateral motion in bipedal walking," in IEEE Int. Conf. Robot. Autom., New Orleans, LA, 2004, pp. 3049-3054.

[13] E. R. Westervelt, G. Buche, and J. W. Grizzle, "Experimental validation of a framework for the design of controllers that induce stable walking in planar bipeds," Int. J. Robot. Res., vol. 23, no. 6, pp. 559-582, 2004

[14] S. Grillner, "Neurobiological bases of rhythmic motor acts in vertebrates," Science, vol. 228, pp. 143-149, 1985.

[15] H. Miura and I. Shimoyama, "Dynamical walk of biped locomotion," Int. J. Robot. Res., vol. 3, no. 2, pp. 60-74, 1984.

[16] K. Hirai, M. Hirose, and T. Takenaka, "The development of Honda humanoid robot," in Proc. 1998 IEEE Int. Conf. Robot. Autom., pp. 160 165

[17] K. Nagasaka, M. Inaba, and H. Inoue, "Stabilization of dynamic walk on a humanoid using torso position compliance control," in Proc. 17th Annu. Conf. Robot. Soc. Japan, 1999, pp. 1193-1194.

[18] T. Sugihara and Y. Nakamura, "Whole-body cooperative COG control through ZMP manipulation for humanoid robots," in IEEE Int. Conf. Robot. Autom., Washington, DC, 2002, pp. 1404-1409.

[19] S. Kajita, F. Kanehiro, K. Kaneko, K. Fujiwara, K. Yokoi, and H. Hirukawa, "Biped walking pattern generation by a simple threedimensional inverted pendulum model," $A d v$. Robot., vol. 17, no. 2, pp. 131-147, 2004.

[20] G. Cheng, S. Hyon, J. Morimoto, A. Ude, J. G. Hale, G. Colvin, W. Scroggin, and S. C. Jacobsen, "CB: A humanoid research platform for exploring neuroscience," Adv. Robot., vol. 21, no. 10, pp. 1097-1114, 2007

[21] S. H. Strogatz, Nonlinear Dynamics and Chaos. Reading, MA: Addison-Wesley, 1994.

[22] N. Yamasaki, M. Kawachi, T. Nishizawa, T. Suzuki, and A. Kusumoto, Encyclopedia of Foot (in Japanese). Tokyo: Asakura Shoten, 1999.

[23] J. Perry, Gait Analysis: Normal and Pathological Function. Thorofare, NJ: Slack Inc., 1992.

[24] D. A. Winter, Biomechaniics and Motor Control of Human Movement, 2nd ed. Hoboken, NJ: Wiley, 1990.

[25] J. Morimoto and C. G. Atkeson, "Minimax differential dynamic programming: An application to robust biped walking," in Advances in Neural Information Processing Systems 15, S. Becker, S. Thrun, and K. Obermayer, Eds. Cambridge, MA: MIT Press, 2003, pp. 1563 1570 .

[26] J. Morimoto and C. G. Atkeson, "Learning biped locomotion: Application of poincaré-map-based reinfrocement learning," IEEE Robot. Autom. Mag., vol. 14, no. 2, pp. 41-51, Jun. 2007.

\section{Multisensor Input for CPG-Based Sensory-Motor Coordination}

\author{
R. Héliot and B. Espiau
}

Abstract-This paper describes a method for providing in real time a reliable synchronization signal for cyclical motions such as steady-state walking. The approach consists in estimating online a phase variable on the basis of several implicit central pattern generator associated with a set of sensors. These sensors can be of any kind, provided their output strongly reflects the timed motion of a link. They can be, for example, spatial position or orientation sensors, or foot sole pressure sensors. The principle of the method is to use their outputs as inputs to nonlinear observers of modified Van der Pol oscillators that provide us with several independent estimations of the overall phase of the system. These estimations are then combined within a dynamical filter constituted of a Hopf oscillator. The resulting phase is a reliable indexing of the cyclic behavior of the system, which can finally be used as input to low-level controllers of a robot. Some results illustrate the efficiency of the approach, which can be used to control robots.

Index Terms-Central pattern generator (CPG), oscillator, sensors, sensory-motor coordination.

\section{INTRODUCTION}

A classical way of generating cyclic motions for articulated systems is to synthesize a rhythm generator, called central pattern generator (CPG). The CPG concept comes from biology [1], [2]; it is a small neural network, located at the spinal level, able to generate rhythmic commands for the muscles. CPGs receive inputs from higher parts of the central nervous system, and also from peripheral afferents; thus, its functioning results from an interaction between central commands and local reflexes. The implementation of this artificial CPG is usually achieved either by designing and training an adequate artificial neural network [3], or by using explicitly nonlinear differential equations acting as an adaptable dynamical oscillator [4]. This concept allows us to easily achieve coordination between different limbs, using independent oscillators for each limb, that are coupled together. In that case, phase oscillators are often used in order to easily model the interaction between two oscillators [5], [6].

When several actuators that need to be synchronized are considered, e.g., for multilegged robots, multiple or coupled oscillators can be used, generally under the control of a master CPG. For this kind of classical CPG-based approach, the literature is quite extensive [4], [7]-[9]. However, the need for adaptation of the system to environmental changes, external requirements, or proprioceptive information through sensory signals is more rarely addressed. We can nevertheless refer the reader to a few recent papers in the field that give a good idea of the state of the art; in [10], a robot fish can avoid obstacles through infrared sensors, the output of that will allow the system to select CPG model strategies among a set of predefined ones. Fukuoka et al. [11] modulate the phase of a neural oscillator on the basis of the

Manuscript received January 31, 2007; revised October 11, 2007. This paper was recommended for publication by Associate Editor A. Takanisi and Editor F. Park upon evaluation of the reviewers' comments.

R. Héliot is with the Commissariat Energie Atomique-Laboratoire d'Electronique et de Technologie de l'Information (CEA-LETI), 38000 Grenoble Cedex, France. He is also with the Institut National de Recherche en Informatique et en Automatique (INRIA) Rhône Alpes, 38334 Saint Ismier Cedex, France (e-mail: rodolphe.heliot@inrialpes.fr).

B. Espiau is with the Institut National de Recherche en Informatique et en Automatique (INRIA) Rhône Alpes, 38334 Saint Ismier Cedex, France (e-mail bernard.espiau@inria.fr).

Digital Object Identifier 10.1109/TRO.2008.915433 